\title{
Genomic imprinting of DIO3, a candidate gene for the syndrome associated with human uniparental disomy of chromosome 14
}

\author{
Maria Elena Martinez ${ }^{1}$, David F Cox ${ }^{2}$, Brian P Youth ${ }^{2}$ and Arturo Hernandez ${ }^{\star, 1}$ \\ Individuals with uniparental disomy of chromosome 14 (Temple and Kagami-Ogata syndromes) exhibit a number of \\ developmental abnormalities originating, in part, from aberrant developmental expression of imprinted genes in the DLK1-DIO3 \\ cluster. Although genomic imprinting has been reported in humans for some genes in the cluster, little evidence is available \\ about the imprinting status of $D I O 3$, which modulates developmental exposure to thyroid hormones. We used pyrosequencing \\ to evaluate allelic expression of DLK1 and DIO3 in CDNAs prepared from neonatal foreskins carrying single-nucleotide \\ polymorphisms (SNPs) in the exonic sequence of those genes, and hot-stop PCR to quantify DIO3 allelic expression in cDNA \\ obtained from a skin specimen collected from an adult individual with known parental origin of the DIO3 SNP. In neonatal skin, \\ DLK1 and DIO3 both exhibited a high degree of monoallelic expression from the paternal allele. In the adult skin sample, the \\ allele preferentially expressed is that inherited from the mother, although a different, larger DIO3 mRNA transcript appears the \\ most abundant at this stage. We conclude that $D I O 3$ is an imprinted gene in humans, suggesting that alterations in thyroid \\ hormone exposure during development may partly contribute to the phenotypes associated with uniparental disomy of \\ chromosome 14.
}

European Journal of Human Genetics (2016) 24, 1617-1621; doi:10.1038/ejhg.2016.66; published online 22 June 2016

\section{INTRODUCTION}

Genomic imprinting (GI) refers to an epigenetic modification of the DNA that results in differential gene expression between both alleles depending on their parental origin. ${ }^{1,2}$ GI originates in germ cells, where DNA is differentially methylated between sexes in critical genomic regions of imprinted loci. After fertilization, these differential epigenetic marks are maintained and ultimately translated into preferential allelic expression from one of the alleles. ${ }^{1}$ Although the evolutionary etiology for GI is unclear, ${ }^{2}$ it is a phenomenon that occurs in many mammals, but affects only a small subset of genes. However, appropriate expression of these imprinted genes appears critical for proper developmental and physiological outcomes. In humans, abnormal GI, typically due to mutations and epimutations in regions of the imprinted domain that regulate allele-specific expression, leads to severe phenotypes, like those associated with Prader-Willi, Angelman and Beckwith-Wiedemann syndromes. ${ }^{3,4}$ Altered expression of imprinted genes also occurs in patients with uniparental disomy (UPD) of whole chromosomes or chromosomal regions containing imprinted loci. These individuals carry the same set of epigenetic marks in both copies of the imprinted domains leading to aberrant allelic expression of the genes in the cluster resulting in abnormal developmental and physiological outcomes. ${ }^{5}$

The imprinted DLK1-DIO3 region is located in the distal arm of human chromosome $14 .{ }^{6}$ Patients with maternal or paternal UPD of human chromosome 14 (UPD14; named Temple and Kagami-Ogata syndromes, respectively) present with distinct developmental abnormalities, including hydrocephalus, hypotonia, abnormal growth, mental retardation, craniofacial dismorphisms, altered puberty onset, abnormal rib cage and others. ${ }^{7,8}$ Several genes within this region, including DLK1 and $M E G 3$, are subject to genomic imprinting, that is, they exhibit a high degree of monoallelic expression. ${ }^{6}$ However, it is uncertain whether DIO3 is imprinted. Studies on the placentas of infants with UPD of chromosome 14 and related epimutations suggest that DIO3 is not imprinted in this tissue in humans. ${ }^{9}$ In the mouse, Dio3 is considered an imprinted gene, as fetal tissues exhibit marked preferential Dio3 expression from the paternal allele. ${ }^{10,11}$ In the mouse placenta, Dio3 is preferentially expressed from the paternal allele, although the contribution of the maternal allele to overall Dio3 expression is significant. ${ }^{12}$ We thus hypothesized that human DIO3, like its mouse homolog, is also imprinted in certain issues. Here we report that this is the case in skin.

\section{MATERIALS AND METHODS}

Collection of human samples, processing and analysis

Foreskin samples were collected from neonatal circumcisions performed by the Department of Pediatrics Newborn Service at Maine Medical Center (Portland, ME, USA). No personal information was collected from these infants. For a set of 14 samples, a protocol for research on Human Subjects was approved by the Internal Review Board at Maine Medical Center. For this set of samples, parental consent was obtained and, in addition to a foreskin specimen from the infant, buccal swabs were collected from the mother or both parents for genotypic analysis. No personal or clinical information was collected in relationship with these infants. In addition, an adult skin specimen

${ }^{1}$ Center for Molecular Medicine, Maine Medical Center Research Institute, Scarborough, ME, USA; ${ }^{2}$ Department of Pediatrics, Maine Medical Center, Portland, ME, USA *Correspondence: Dr A Hernandez, Center for Molecular Medicine, Maine Medical Center Research Institute, 81 Research Drive, Scarborough, ME 04074 , USA. Tel: +1 207396 8139; Fax: +1 207396 8110; E-mail: hernaa@mmc.org

Received 15 December 2014; revised 29 April 2016; accepted 20 May 2016; published online 22 June 2016 
was obtained from a healthy, 41-year-old male volunteer, and buccal swabs from him and his relatives were collected using a kit from Epicentre Technologies (Madison, WI, USA). DNA and total RNA were isolated from the skin specimens using RNAeasy and DNA kits from Qiagen (Germantown, MD, USA). Genotyping of single-nucleotide polymorphisms (SNP; NCBI rs1802710, chr14.hg19:g.101200645T >C and rs945006, chr14.hg19:g.102029277T > G, for the DLK1 and DIO3 genes, respectively) was performed by DNA sequencing at the Protein, Nucleic Acid and Cell Imaging Core at MMCRI and confirmed by pyrosequencing (EpigenDX Hopkinton, MA, USA). Reverse transcription of total RNA (1 ug) was performed with MMLV reverse transcriptase (Life Technologies, Carlsbad, CA, USA) using standard protocols. A genomic DNA DIO3 fragment containing the DIO3 SNP was amplified by PCR from buccal swabs, using taq DNA polymerase (Denville Scientific, South Plainfield, NJ, USA) and primers 5'-GGTTC CCTGTTGCTTTTGTG-3' (forward) and 5'-CCCTCAAGGTTTAGGT GCTG-3' (reverse). DIO3 and DLK1 alelle quantification in cDNAs from foreskin samples was performed by pyrosequencing (EpigenDX Hopkinton). In the cDNA from adult skin, DIO3 allele quantification was performed using a variation of the hop-stop PCR method ${ }^{13}$ (The description and validation of this method are included in Supplementary Figure 1). The data pertaining to the abundance of the SNP variants in the samples studied have been submitted to dsSNP Database at NCBI, and the submission is being processed (http://www.ncbi.nlm.nih.gov/ SNP/snp_viewTable.cgi?handle=MMCRIHERNANDEZ).

Commercial human fetal and adult total RNA was obtained from Origene (Rockville, MD, USA), and subjected to northern blot analysis using standard protocols using as a probe a $0.7 \mathrm{~kb}$ Sal I/Sac I genomic fragment containing exonic DIO3 sequence. This probe was labeled with ${ }^{32} \mathrm{P}$ using ${ }^{32} \mathrm{P}-\mathrm{dCTP}$ (MP Biomedicals, Santa Ana, CA, USA) and the random primer labeling kit from Pfizer (New York, NY, USA). Statistical significance between two groups was determined by the Student's $t$-test. $P<0.05$ was considered significant.

\section{RESULTS}

We genotyped the DLK1 and DIO3 SNPs (GenBank rs1802710 and rs945006, respectively) in 52 neonatal foreskin samples. Genotyping results are shown in Figure 1a. The T/C genotype of the DLK1 SNP was present in $56.1 \%$ of the individuals, whereas the T/G genotype of the DIO3 SNP was identified in $36.4 \%$ of the individuals. According to GenBank databases, the prevalence of the G variant of DIO3 is very low, low and high in individuals of Asian, European and African descent, respectively.

RNA was extracted from seven foreskin samples carrying the informative variation of the DLK1 SNP, and subjected to reverse transcription. The obtained complementary DNA (cDNA) was used as a template for PCR and subsequent allele quantification by pyrosequencing (Figure 1b). All seven samples analyzed exhibited a very high degree $(>95 \%)$ of monoallelic expression for DLK1. This is consistent with the published data on the genomic imprinting of DLK1 in other developing tissues. ${ }^{6}$ Likewise, allelic quantification of DIO3 was performed in eight samples carrying the DIO3 G/T genotype (Figure 1c). On average, the degree of monoallelic expression for $D I O 3$ was $74 \%$ (Figures $1 \mathrm{c}$ and d; Representative pyrograms for DLK1 and DIO3 allele quantification in two of the samples analyzed are shown in Supplementary Figure 2). Allelic contribution to DIO3 expression was significantly biased towards one of the alleles when compared to the allelic contribution to genomic DNA as quantified by the same method (Figure 1d). Mean DIO3 allelic cDNA ratio was higher than 3 , and significantly different to that in genomic DNA (Figure 1e). These results demonstrate strong preferential allelic expression of the human DIO3 gene. To ascertain the parental origin of the preferentially expressed DIO3 allele, we collected an additional number of foreskin specimens from children whose mothers (or both parents) consented to provide a buccal swab for DLK1 and DIO3 genotyping. From these sets of samples, we identified two sets for each of the genes that proved informative as to the parental origin of each allele. Pyrosequencing evaluation of DLK1 and DIO3 cDNAs obtained from these samples confirmed preferential allelic expression in both genes (Figure 1f). On the basis of the mother and/or father genotype we concluded that, in newborn foreskin, both DLK1 and DIO3 are preferentially expressed from the allele inherited from the father.

We also performed DIO3 allelic quantification studies on RNA isolated from a skin biopsy obtained from an adult male volunteer heterozygous for the T/G DIO3 polymorphism. His father and one of his sons were also heterozygous for the T/G DIO3 polymorphism, but not his mother, who was homozygous for the $\mathrm{T}$ variant of $\mathrm{DIO} 3$ (Figure 2a). cDNA containing the DIO3 SNP was prepared from a skin biopsy of this individual (Figure $2 b$ ). Sense and antisense Sanger sequencing of the amplicon indicated the presence of the polymorphisms, with comparable peaks for both variants when the amplicon originated from genomic DNA (Figure 2c, top). However, the T peak (and the A peak for the antisense strand) markedly predominated in the amplicon originated from cDNA (Figure 2c, bottom).

DIO3 allele quantification in this cDNA sample was performed using a variation of the hot-stop PCR method ${ }^{13}$ (see Materials and Methods). Quantification of the contribution of each allele of the subject to the genomic DNA was comparable, but in the cDNA the contribution from the $\mathrm{T}$ allele was much higher than that from the $\mathrm{G}$ allele (Figure $2 \mathrm{~d}$ ). Densitometry quantification in four different replicates indicated an $\sim 50 \%$ presence of each of the variants in genomic DNA, but an $80 \%$ presence of the $\mathrm{T}$ variant (versus $20 \%$ of the $\mathrm{G}$ variant) in the cDNA pool. These results indicate strong preferential expression of DIO3 from the $\mathrm{T}$ allelic variant in this individual. As this variant was inherited from his mother, the results demonstrate that the maternal DIO3 allele is preferentially expressed in the skin of this adult individual. The DLK1 genotype of this sample $(\mathrm{T} / \mathrm{T})$ was not informative about the paternal origin of the DLK1 alleles.

Northern blot analysis of RNA from fetal and adult human skin reveals different abundance of DIO3 mRNA transcripts at these developmental stages. The characterized $2.1-2.2 \mathrm{~kb}$ transcript is the most abundant in fetal skin, but a larger $2.5-2.6 \mathrm{~kb}$ transcript is the only one detected by this method in adult skin (Figure 2f). This observation indicates a marked shift from fetal to adult life in the predominant DIO3 mRNA species in this tissue.

\section{DISCUSSION}

Our results demonstrate that human DIO3 is preferentially expressed from the paternally inherited allele in neonatal foreskins. This is also applicable to DKL1. For both DLK1 and DIO3, there is not a particular polymorphic variant that is preferentially expressed, suggesting that the results observed are truly the result of genomic imprinting and not due to the presence of genetic or epigenetic factors that might be specifically associated to one of the polymorphic variants. These findings are consistent with observations showing preferential allelic expression of the paternal DLK1 allele in human fetal tissues, ${ }^{6}$ and also with those showing that both Dlk1 and Dio3 genes are preferentially expressed from the paternal allele in the mouse fetus. ${ }^{10,11}$

In this context, our additional observation of preferential maternalallele expression of $\mathrm{DIO} 3$ in adult skin is not a fully anticipated result. The most likely explanation is that the larger DIO3 transcript present in the adult skin exhibits opposite imprinting. This larger 
a

Genotype Distribution of DLK1 rs 1802710 and DIO3 rs945006 in the 66 samples collected

\begin{tabular}{lll}
\hline & T/T & $17(25.8 \%)$ \\
DLK1 & T/C & $37(56.1 \%)$
\end{tabular}

C/C $12(18.2 \%)$

$\begin{array}{lll}\text { DIO3 } & \text { T/T } & 41(62.1 \%) \\ \text { T/G } & 24(36.4 \%)\end{array}$

G/G $1(1.5 \%)$

Combined Genotypes

DLK1 DIO3 Number (\%)

$\begin{array}{lll}\mathrm{T} / \mathrm{T} & \mathrm{T} / \mathrm{T} & 14(82 \%) \\ \mathrm{T} / \mathrm{T} & \mathrm{T} / \mathrm{G} & 3(18 \%)\end{array}$

$\mathrm{T} / \mathrm{T} \quad \mathrm{G} / \mathrm{G} \quad 0(0 \%)$

T/C T/T $22(59 \%)$

T/C T/G $14(38 \%)$

T/C G/G $1(3 \%)$

$\mathrm{C} / \mathrm{C} \quad \mathrm{T} / \mathrm{T} \quad 5(42 \%)$

C/C T/G $7(58 \%)$

C/C G/G $0(0 \%)$ b

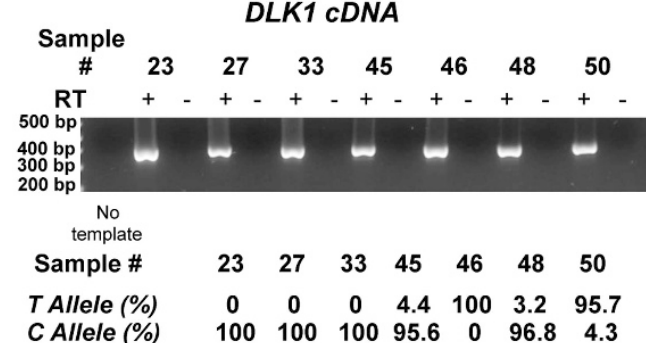

C

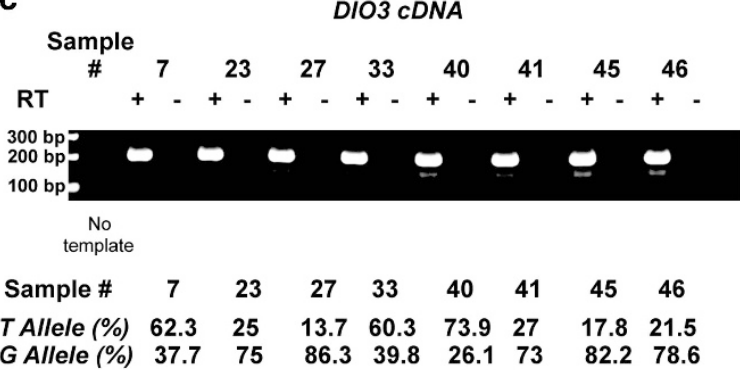

d

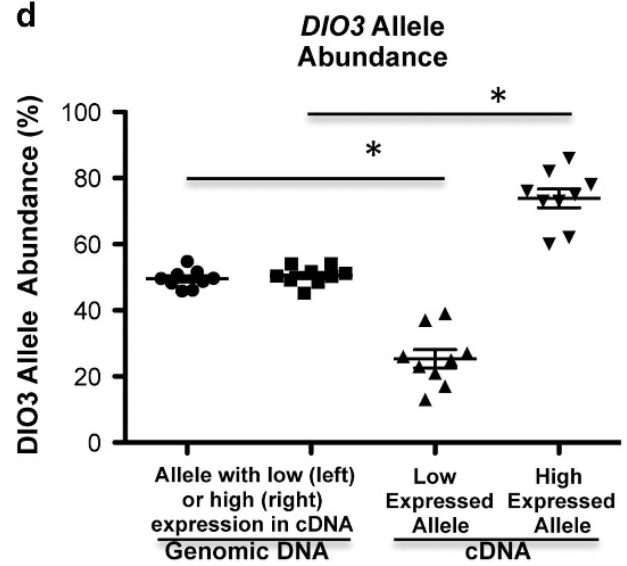

e

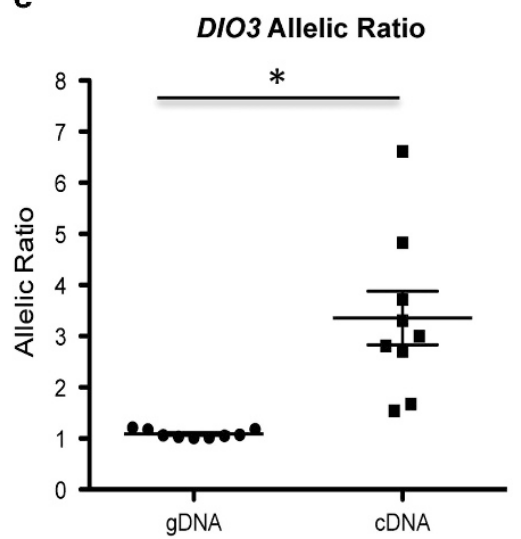

f

\begin{tabular}{cccccc} 
Gene & Sample \# & $\begin{array}{c}\text { Mother } \\
\text { Genotype }\end{array}$ & $\begin{array}{c}\text { Father } \\
\text { Genotype }\end{array}$ & $\begin{array}{c}\text { Infant } \\
\text { Genotype }\end{array}$ & $\begin{array}{c}\text { Foreskin cDNA } \\
\text { Allele quantification }\end{array}$ \\
\hline \multirow{2}{*}{ DLK1 } & 54 & C/C & Unknown & T/C & C (13\%), T (87\%) \\
& 58 & C/C & Unknown & T/C & C (18\%), T (82\%) \\
DIO3 & 62 & T/G & T/T & T/G & G (31\%), T (69\%) \\
& 65 & T/G & T/T & T/G & G (22\%), T (78\%)
\end{tabular}

Figure 1 Preferential allelic expression of DLK1 and DIO3 in human newborn foreskins. (a) Summary of DLK1 and DIO3 SNP genotypes in the samples collected. ( $b$ and $\mathbf{c}$ ) Agarose gels of DLK1 and DIO3 cDNAs in selected samples with its corresponding allelic quantification by pyrosequencing. (d) Pyrosequencing data on the abundance of DIO3 alleles in genomic DNA and mRNA-derived cDNA. (e) Higher abundance/lower abundance DIO3 allelic ratio in genomic DNA and mRNA-derived cDNA. (f) Genotype data and foreskin cDNA allele quantification in the sample sets that were informative as to the parental origin of each allele. ${ }^{*} P<0.01$ as determined by the Student's $t$-test. RT, reverse transcription.

DIO3 mRNA species has been identified in skin cancer cells, and appears to be transcribed by an unidentified upstream promoter. ${ }^{14}$ It is thus possible that different epigenetic factors control its transcription. Furthermore, preferential expression from the maternal Dio3 allele has been described in the adult rat hippocampus, ${ }^{15}$ a tissue in which the larger Dio3 transcript accounts for the vast majority of Dio3 expression. ${ }^{16}$ It is thus plausible that the larger DIO3 transcript, more abundant in adult tissues, ${ }^{17}$ exhibits an allelic pattern of expression that is opposite to that observed for the shorter, development-associated Dio3 transcript. Opposite imprinting between transcripts from the same gene, or as a result of different developmental stages, has been observed for other imprinted genes. ${ }^{18-20}$ In this regard, we have recently described tissue-, developmental stage- and transcript-specific variations in the allelic expression of Dio3 in the mouse. ${ }^{21}$ Recently, Kagami et al. ${ }^{22}$ have not found sufficient evidence of DIO3 imprinting when analyzing human placental tissue which, as in the mouse, expresses exclusively the short $\mathrm{DIO} 3$ transcript. $^{23}$ That result is probably due to a low or inexistent degree of preferential allelic expression of DIO3 in the human placenta, a finding that is consistent with the very relaxed pattern of Dio3 imprinting observed in the same tissue of the mouse. $^{12}$ 
a

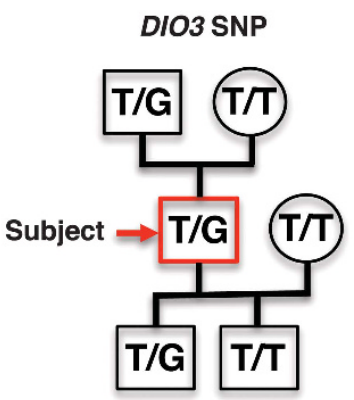

d

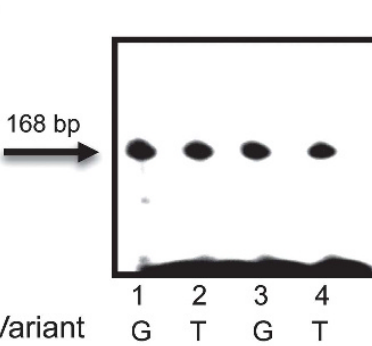

Template $\overline{\text { Genomic DIO3 }}$

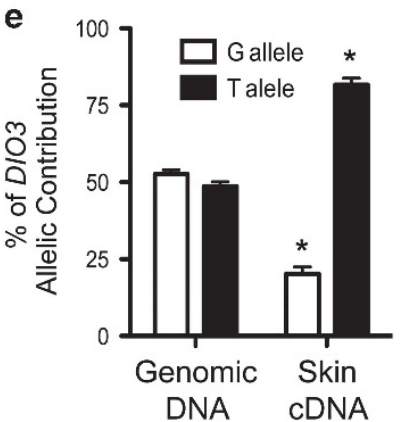

b

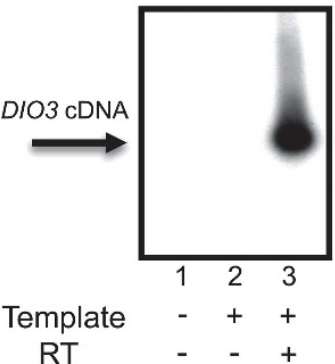

bp

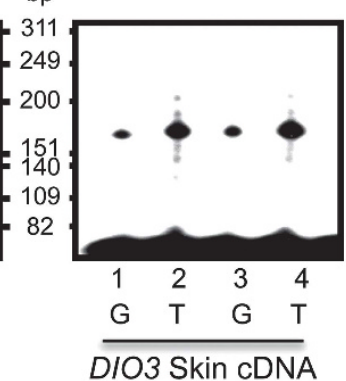

c DIO3 Genomic DNA

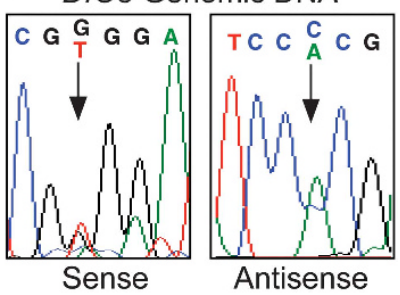

DIO3 CDNA

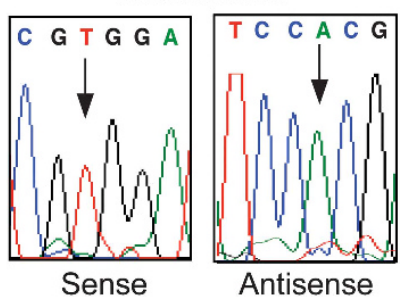

f

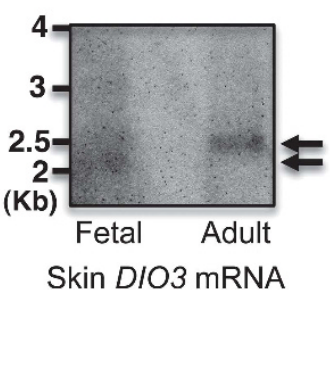

Figure 2 DIO3 imprinting in adult human skin. (a) Pedigree of the subject (highlighted in red) regarding the single-nucleotide polymorphism and DIO3 rs945006; (b) mRNA-specific origin of the DIO3 cDNA fragment analyzed; (c) Sequence of DIO3 rs945006 in the genomic DNA and in RNA-derived cDNA from the adult skin sample; (d) Agarose gels of allele-specific DIO3 PCR products from genomic DNA and cDNA from the adult skin sample. (e) Quantification of the results shown in $\mathbf{d}$; (f) Northern blot analysis of human fetal and adult skin mRNA. Arrows indicate the two transcript that are most abundant at each age. ${ }^{*} P<0.01$ as determined by the Student's $t$-test $(n=4)$.

The genomic imprinting of DIO3 suggest that abnormal thyroid hormone levels may occur in patients with Temple and Kagami-Ogata syndromes, but no such abnormalities have been reported. Observations in a mouse model of genetic inactivation of the paternal Dio3 allele, a model comparable to Temple syndrome in humans, indicate elevated levels of thyroid hormone at neonatal stages, ${ }^{21}$ but not in adulthood. ${ }^{24}$ However, owing to timing differences in brain developmental processes, the hypothalamic-pituitary-thyroid axis is already mature in human newborns, whereas it is still immature in newborn rodents. It is thus possible that abnormal thyroid hormone exposure occurs in utero in patients with the above syndromes.

Our results demonstrate that the human DIO3 is an imprinted gene preferentially expressed from the paternal allele in neonatal skin, and suggest that DIO3 imprinting is transcript-specific. Thus, abnormal thyroid hormone exposure of developing tissues as a result of altered thyroid hormone metabolism may contribute to the phenotypic anomalies observed in Temple and Kagami-Ogata syndromes. Our results warrant additional studies on the genomic imprinting status of DIO3 in other developing human tissues, especially those relevant to those syndromes.

\section{CONFLICT OF INTEREST}

The authors declare no conflict of interest.

\section{ACKNOWLEDGEMENTS}

We are grateful to Donald St Germain for critical reading of the manuscript and to R. Calvo, D. Collison, D. Burris and I. Emery for assistance with the collection of the samples. This study was partially supported by the MMC BioBank, a core facility of Maine Medical Center Research Institute, and by grants from the National Institutes of Mental Health and Diabetes, Digestive and Kidney Diseases (MH083220, DK095908 and MH096050). In this work, we used the facilities of the Protein, Nucleic Acid and Cell Imaging Core at Maine Medical Center Research Institute, supported by NIH grant P30 GM103392.

\footnotetext{
1 Reik W, Walter J: Genomic imprinting: parental influence on the genome. Nat Rev Genet 2001; 2: 21-32.

2 Kelsey G: The hows and whys of imprinting. Trends Genet 2000; 16: 15-16.

$3 \mathrm{Knoll} \mathrm{JH}$, Nicholls RD, Magenis RE, Graham Jr JM, Lalande M, Latt SA: Angelman and Prader-Willi syndromes share a common chromosome 15 deletion but differ in the parental origin of the deletion. Am J Med Genet 1989; 32: 285-290.
} 
4 Maher ER, Reik W: Beckwith-Wiedemann syndrome: imprinting in clusters revisited. J Clin Invest 2000; 105: 247-252.

5 Preece MA, Moore GE: Genomic imprinting, uniparental disomy and foetal growth. Trends Endocrinol Metab 2000; 11: 270-275.

6 Wylie AA, Murphy SK, Orton TC, Jirtle RL: Novel imprinted DLK1/GTL2 domain on human chromosome 14 contains motifs that mimic those implicated in IGF2/H19 regulation. Genome Res 2000; 10: 1711-1718.

7 Buiting K, Kanber D, Martin-Subero Jl et al: Clinical features of maternal uniparental disomy 14 in patients with an epimutation and a deletion of the imprinted DLK1/GTL2 gene cluster. Hum Mutat 2008; 29: 1141-1146.

8 Cotter PD, Kaffe S, McCurdy LD, Jhaveri M, Willner JP, Hirschhorn K: Paterna uniparental disomy for chromosome 14: a case report and review. [Review] [27 refs]. Am J Med Genet 1997; 70: 74-79.

9 Hosoki K, Kagami M, Tanaka T et al: Maternal uniparental disomy 14 syndrome demonstrates prader-willi syndrome-like phenotype. J Pediatr 2009; 155: 900-903e1.

10 Tsai CE, Lin SP, Ito M, Takagi N, Takada S, Ferguson-Smith AC: Genomic Imprinting contributes to thyroid hormone metabolism in the mouse embryo. Curr Biol 2002; 12: 1221-1226.

11 Hernandez A, Fiering S, Martinez E, Galton VA, St Germain D: The gene locus encoding iodothyronine deiodinase type 3 (Dio3) is imprinted in the fetus and expresses antisense transcripts. Endocrinology 2002; 143: 4483-4486.

12 Lin SP, Coan P, da Rocha ST et al: Differential regulation of imprinting in the murine embryo and placenta by the DIk1-Dio3 imprinting control region. Development 2007; 134: 417-426.

13 Uejima H, Lee MP, Cui H, Feinberg AP: Hot-stop PCR: a simple and general assay for linear quantitation of allele ratios. [erratum appears in Nat Genet 2001;28(1):97]. Nat Genet 2000; 25: 375-376.

14 Dentice M, Luongo C, Huang S et al: Sonic hedgehog-induced type 3 deiodinase blocks thyroid hormone action enhancing proliferation of normal and malignant keratinocytes. Proc Natl Acad Sci USA 2007; 104: 14466-14471.
15 Sittig LJ, Herzing LB, Shukla PK, Redei EE: Parent-of-origin allelic contributions to deiodinase-3 expression elicit localized hyperthyroid milieu in the hippocampus. Mol Psychiatry 2011; 16: 786-787.

16 Tu HM, Legradi G, Bartha T, Salvatore D, Lechan R, Larsen PR: Regional expression of the type 3 iodothyronine deiodinase messenger ribonucleic acid in the rat central nervous system and its regulation by thyroid hormone. Endocrinology 1999; 140: 784-790.

17 Hernandez A, Martinez E, Croteau W, St Germain D: Complex organization and structure of sense and antisense transcripts expressed from the DIO3 gene imprinted locus. Genomics 2004; 83: 413-424.

18 Hayward BE, Kamiya M, Strain L et al: The human GNAS1 gene is imprinted and encodes distinct paternally and biallelically expressed G proteins. Proc Natl Acad Sci USA 1998; 95: 10038-10043.

19 Wroe SF, Kelsey G, Skinner JA et al: An imprinted transcript, antisense to Nesp, adds complexity to the cluster of imprinted genes at the mouse Gnas locus. Proc Natl Acad Sci USA 2000; 97: 3342-3346.

20 Arnaud $\mathrm{P}$, Monk D, Hitchins $\mathrm{M}$ et al: Conserved methylation imprints in the human and mouse GRB10 genes with divergent allelic expression suggests differential reading of the same mark. Hum Mol Genet 2003; 12: 1005-1019.

21 Martinez ME, Charalambous M, Saferali A et al: Genomic imprinting variations in the mouse type 3 deiodinase gene between tissues and brain regions. $\mathrm{Mol}$ Endocrinol 2014; 28: 1875-1886.

22 Kagami M, Matsuoka K, Nagai T et al: Paternal uniparental disomy 14 and related disorders: placental gene expression analyses and histological examinations. Epigenetics 2012; 7: 1142-1150.

23 Huang SA, Dorfman DM, Genest DR, Salvatore D, Larsen PR: Type 3 iodothyronine deiodinase is highly expressed in the human uteroplacental unit and in fetal epithelium. J Clin Endocrinol Metab 2003; 88: 1384-1388.

24 Ueta CB, Oskouei BN, Olivares EL et al: Absence of myocardial thyroid hormone inactivating deiodinase results in restrictive cardiomyopathy in mice. Mol Endocrinol 2012; 26: 809-818.

Supplementary Information accompanies this paper on European Journal of Human Genetics website (http://www.nature.com/ejhg) 